\title{
Coulisses
}

Revue de théâtre

43 | Automne 2011

Reviviscences de Faust

\section{Edmond Rostand entre Faust et Don Juan}

\section{Bertrand Degott}

\section{CpenEdition}

Journals

Édition électronique

URL : https://journals.openedition.org/coulisses/362

DOI : $10.4000 /$ coulisses.362

ISSN : 2546-9460

\section{Éditeur}

Presses universitaires de Franche-Comté

\section{Édition imprimée}

Date de publication : 31 décembre 2011

Pagination : 120-126

ISBN : 978-2-84867-404-9

ISSN : 1150-594X

Référence électronique

Bertrand Degott, " Edmond Rostand entre Faust et Don Juan », Coulisses [En ligne], 43 | Automne 2011, mis en ligne le 30 novembre 2016, consulté le 29 décembre 2022. URL : http:// journals.openedition.org/coulisses/362 ; DOI : https://doi.org/10.4000/coulisses.362 


\title{
Edmond Rostand entre Faust et Don Juan
}

\author{
Bertrand Degott
}

1 Il est communément admis que, dramatiquement parlant, passé le triomphe de Cyrano de Bergerac (1897) Rostand n'a cessé d'écrire en dessous de lui. Au terme d'un parcours difficile, encore assombri par la guerre, il laisse inachevés son adaptation du premier Faust de Goethe et La Dernière Nuit de Don Juan. Pour la bonne intelligence de la suite de notre étude, il convient de résumer la fable de ce " poème dramatique en deux parties et un prologue ». Dans le prologue (48 vers), Don Juan, que la Statue du Commandeur traîne en Enfer, ne se sent pas sitôt happé par la Griffe du Diable qu'il lui demande et obtient d'elle dix ans de sursis. Les deux parties de la pièce (1017 vers) ont lieu dans un palais à Venise. Les dix ans sont écoulés; alors que Don Juan se prépare pour le bal, il laisse entrer un montreur de marionnettes qui se révèle être le Diable. Celui-ci le rappelle à ses engagements, mais lui promet de ne l'emporter que vaincu. Commence alors, comme une partie de cartes, une lutte qui ne s'achèvera que lorsque Don Juan aura admis qu'il n'a rien créé. Il n'a plus qu’à rentrer à son tour dans le petit théâtre et à devenir pour l'éternité sa propre marionnette.

2 Même s'il est tentant d'associer les deux œuvres et d'écrire avec Caroline de Margerie que « Faust et Don Juan sont des pièces d'où l'espoir s'est enfui " ${ }^{1}$, cela n'est possible qu'à condition d'escamoter les composantes génériques qui distinguent une traduction, fûtelle adaptation versifiée, d'une œuvre de création. Tout porte à croire du reste que durant ces années noires Rostand n'a vraiment travaillé qu'à celle-ci, se contentant d'annoncer celle-là, mais promettant l'une et l'autre, tour à tour accréditant et étouffant les rumeurs. Or l'œuvre théâtrale est en vers, c'est dire que Rostand est aussi et avant tout poète. Parallèlement à ces deux œuvres dramatiques, il écrit des poèmes très souvent des sonnets -, qu'il publie au gré des revues. Ceux-ci constitueront la matière de deux recueils posthumes, Le Vol de la Marseillaise (1919) et Le Cantique de l'aile (1922). C'est dans ce dernier pan de l'œuvre que nous nous proposons d'étudier la présence de Faust - sa reviviscence si l'on veut, sa résurgence du moins. En effet, si peu que Rostand se soucie de sa traduction durant cette période, le personnage de Faust et la matière même de la pièce de Goethe ne cessent pour autant de l'inspirer et d'animer 
sa création propre. Sans doute les circonstances historiques n'y jouent-elles pas un rôle moindre que sa mythologie personnelle, mais on peut tout au moins tenter de cerner leur part respective. On a pu écrire de Cyrano de Bergerac que « cette pièce se passe dans le Lagarde et Michard $»^{2}$. Plus généralement encore, l'œuvre de Rostand ne cesse de réfléchir la littérature et à plus forte raison de se réfléchir elle-même. Ce qu'il en est alors des réticences de Rostand vis-à-vis de Faust - du mythe tel que l'a transmis Goethe et de sa propre traduction de l'«Ur-Faust »-, la lecture de ses poésies et surtout de La Dernière Nuit de Don Juan nous permettront d'en apprendre davantage.

\section{Qu'il y a achèvement et achèvement}

3 La traduction de Faust par Edmond Rostand a tout d'une Arlésienne : on en parle sans cesse, on la dit achevée, mais on ne la voit guère... Selon Rosemonde, qui date de 1900 l'arrivée d'Edmond au pays basque, elle "fut terminée vers cette époque ». Le problème est d'une part qu'elle en dit autant de poèmes qui dans Les Musardises et Le Cantique de l'Aile sont datés entre 1891 et 1905 et d'autre part qu'elle ne cite que deux passages de cette "magistrale traduction... si lyriquement fidèle $»^{4}$. La rumeur, les journaux et la correspondance y font d'ailleurs constamment référence. En mars 1903, Coquelin s'excuse auprès de Rostand d'avoir accrédité un faux bruit: «à propos de Faust, il y a un journal de Vienne qui m'a fait dire par la plume de $\mathrm{M}$. Lothar que vous le faisiez pour moi - alors que je lui ai dit simplement ceci : on a parlé d'un Faust il y a 3 ou 4 ans, il en était question chez Sarah et puis je n'en ai plus entendu parler $»^{5}$. Cinq ans plus tard, en février 1908, L'Écho de Paris annonçant que Faust va être joué, Rostand dément par télégramme : la pièce est écrite certes, mais sa priorité est Chantecler ${ }^{6}$. À n'en pas douter certaines scènes sont alors achevées, celle de l'écolier notamment, comme en témoigne une lettre de Sarah : «Richepin m'en a apporté un [de Faust] en vers et je l'ai refusé parce que j'avais encore dans l'oreille les vers que vous m'aviez dits sur la scène de l'écolier " ${ }^{7}$. Dans l'interview qu'il donne au Gil Blas en janvier 1913, Rostand fait remonter le plus clair de son travail aux années 1890:

Faust ?... Ce n'est pas une œuvre récente. Faust est antérieur à Cyrano. [...] Pour ma part, je n'ai voulu qu'adapter simplement la première partie, en suivant le texte original, le plus possible. À ce moment-là, je venais de terminer mes études et je savais parfaitement l'allemand. Je me suis toujours refusé à mélanger les deux Faust si distincts et si particuliers ${ }^{8}$.

4 Mais quant aux progrès de la pièce il n'est pas autrement précis. Et s'il se refuse à la faire jouer, ce n'est plus alors parce qu'une autre a la priorité, mais par « ennui du travail matériel »', au nom de la liberté de l'artiste. Faute d'un manuscrit qui l'infirme, il faut cependant admettre que Rostand n'a jamais achevé son adaptation en vers. Et même s'il considère qu'avec son édition en 2007 «ce Faust pourra enfin être lu et joué $»^{10}$, Philippe Bulinge n'a pu fournir qu'un texte incomplet et fautif. Nos réserves vis-à-vis de son édition s'autorisent de motifs non seulement génétiques mais également métriques: l'éditeur établit son texte à partir de sources aussi variées qu'incertaines et surtout témoigne ce faisant d'une grande méconnaissance du vers rostandien.

5 Les déclarations concernant La Dernière Nuit de Don Juan sont pareillement contradictoires. Dans la même interview de janvier 1913, Rostand en parle ainsi :

C'est une sorte de poème théâtral assez long, d'une coupe singulière, qui serait fait

pour tenir l'affiche avec une autre pièce, comme Salomé à l'Opéra - et sans entractes 
ou presque. La Dernière Nuit de Don Juan... une vieille chose encore! Il y a quinze ou seize ans environ que je l'ai écrite. Je l'ai donnée à Le Bargy qui l'aime, qui veut la jouer et qui la jouera la saison prochaine. Vous vouliez savoir mes projets? En voilà un. Il est certain ${ }^{11}$.

Pourtant, quelques mois plus tard, dans une lettre où il se plaint à sa sœur de ses crises de neurasthénie, il réévalue non seulement son projet, mais encore la forme de l'œuvre :

Je n'irai pas à Paris tant que je ne serai pas solide. D'ailleurs D[on] J[uan] n'a aucune importance, ce n'est qu'un acte plutôt fait pour être imprimé, et plutôt que de m'épuiser pour une bluette, je l'éditerai ${ }^{12}$.

7 La pièce «sans entractes ou presque » n'est plus " qu'un acte ». Lorsqu'elle paraît en 1921, un avertissement liminaire nous dit l'état d'inachèvement où Rostand l'avait laissée : « les deux parties de cette pièce étaient entièrement écrites avant la guerre », alors que «le prologue, reconstitué sur des brouillons fragmentaires très raturés, ne peut être considéré que comme une ébauche ${ }^{13}$. Ainsi, de l'aveu même des éditeurs, le prologue n'est pas entièrement de la plume de Rostand. Cependant, Rosemonde et Maurice avaient du vers une science assez sûre pour donner le change car les 48 alexandrins du prologue forment une suite cohérente d'un point de vue dramatique autant que syntaxique et métrique.

Quant au corps de la pièce, à condition de compter pour rien les didascalies rajoutées entre crochets, la seule trace d'inachèvement se trouve à la couture des deux parties. À la fin de la première, on laisse Don Juan, après sept tentatives infructueuses, s'épuiser à chercher derrière les masques l'identité des femmes qu'il a cru posséder et que les mille et trois morceaux de sa liste déchirée ont fait arriver en gondole. Cette seconde ellipse dramatique justifie donc parfaitement une solution de continuité entre les deux parties. Sur le plan métrique, comme la $\mathrm{I}^{\text {re }}$ partie finit sur la rime masculine recommençait:: c'est (p. 82), on attend une rime féminine. Or le vers 580, ouvert par une réplique muette de Don Juan, se réduit à L'Aurore, qui rime avec encore au vers suivant (p. 85). Dans un carnet que Jean Rostand avait en sa possession à la fin des années 1960, on lit pourtant les vers suivants, de la main d'Edmond:

DJ D

[Pas une... Elles sont là toutes... - Toutes...] L'Aurore

Va-t-elle te trouver cherchant la femme encore,

Diogène de pourpre au flambeau de vermeil?

$\mathrm{J}$

- Oh !... et dans tous ces bras, j'ai connu le sommeil !

9 Si la part du vers entre crochets - principalement constituée par la réplique de Don Juan - manque à l'édition de 1921, peut-être les éditeurs ont-ils jugé qu'elle enchaînait mal sur les derniers vers de la première partie ; peut-être aussi ont-ils utilisé d'autres manuscrits, où Rostand avait abandonné cette version. Quoi qu'il en soit, si la mère et le fils n'ont pas fait pour la traduction de Faust ce qu'ils ont fait pour La Dernière Nuit de Don Juan, tout laisse à croire que les conditions matérielles nécessaires n'étaient pas réunies.

\section{Actes et pactes}

En dépit du changement onomastique (du dom portugais au don espagnol), La Dernière Nuit de Don Juan propose une suite au Dom Juan de Molière. Dans le prologue, on entend 
même Sganarelle hurler encore «Mes gages!» (p.14). Pourtant, la vengeance du convive de pierre tourne aussitôt court : à l'instant même où ce représentant de la justice divine fait grâce au séducteur, celui-ci se sent happé par la Griffe du Diable et tout se passe alors comme si, sous cette nouvelle autorité, Molière ne pouvait que passer le relais à Goethe. Arguant qu'« il [lui] reste là-haut pas mal de mal à faire », Don Juan demande à la Griffe un délai de dix ans. C'est alors l'occasion d'un premier pacte, au cours duquel Faust se trouve convoqué tout à la fois comme précédent et comme repoussoir :

C'est la peine avec moi, Griffe, de faire un pacte. Je suis celui qui fait le plus commettre l'Acte,

Le meilleur rabatteur de votre chasse. Et puis,

- Allons, voyons, laissez ce manteau ! - moi, je suis

Autre chose qu'un docteur Faust, qui ne demande

Qu'une bonne petite ouvrière allemande,

Et qui, navré d'avoir, le sot, fait un enfant,

Appelle au dénouement l'Ange qui le défend ! (p. 16)

11 Rappelons que le texte est peu sûr puisqu'il n'a été établi qu'à partir de brouillons. Il n'y a pourtant rien d'incohérent à ce que sous la plume de Rostand le séducteur des mille e tre (même s'il n'a "sur [s]a liste, encor, que peu de noms ») disqualifie le pacte faustien sur son objet même: la rime Acte:: pacte propose sa réévaluation, loin des projets bourgeois prêtés au docteur, sur la base d'une idéalisation diabolique, c'est-à-dire subvertie, du sexe.

Ce pacte initial fait l'objet d'une reconduction burlesque au cours de la première partie. En effet, c'est en badinant avec la Marionnette du Diable que Don Juan conclut un second marché :

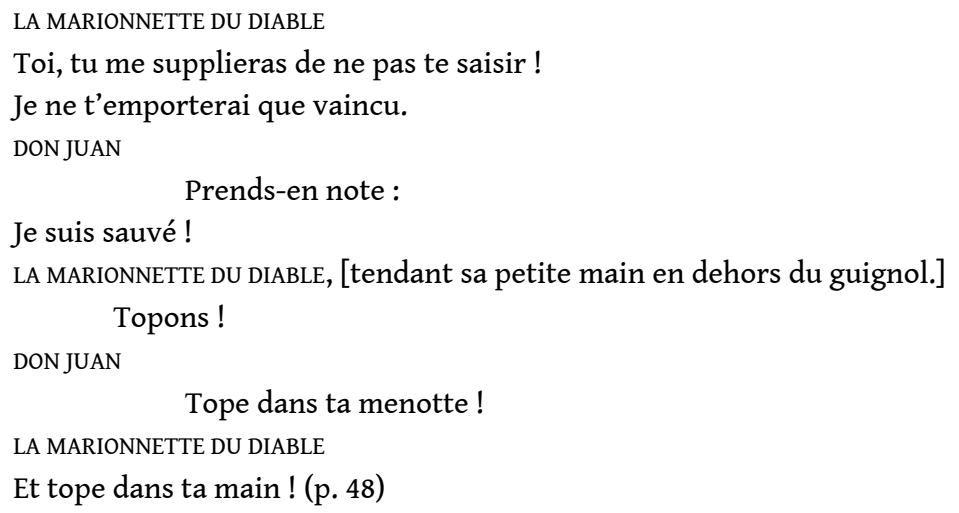

13 Conduite sur le mode ludique, la scène n'en constitue pas moins le véritable nœud de la pièce. Comme toujours sur la scène dramatique, la théâtralité procède à un renversement de l'illusion et le jeu dans le jeu favorise le dévoilement. Sitôt ce pacte conclu dans le cadre du castelet, la marionnette disparaît et le Diable se fait reconnaître : c'est lui le vieux montreur de marionnettes! Son rôle consistera dès lors à défaire une à une les illusions de Don Juan, jusqu'à le " décortiqu[er] de [s]a dernière écorce » (p. 136). Ce Diable montreur de marionnettes n'est d'ailleurs pas sans évoquer le Méphistophélès qui offre à Faust l'intermède du "Walpurgisnachtstraum ». "Par ici : ma parole, un vrai petit théâtre!» (p. 90), lui lance-t-il dans la traduction de Rostand. On ne peut s'empêcher d'associer à ce " petit théâtre " l'idée d'un spectacle de guignol. Réduit in fine à une marionnette, Don Juan n'en demeure pas moins «celui qui fait le 
plus commettre l'[a]cte ", à cette nuance près que dépouillé de sa majuscule acte n'a plus désormais que son sens théâtral...

\section{Trödelhexe}

Le Vol de la Marseillaise rassemble des poésies de circonstance écrites durant la Première Guerre mondiale. Elles sont rassemblées à la mort de Rostand, dans un ordre qui est grosso modo celui de leur publication dans des périodiques tels que Le Figaro, Le Gaulois, Les Annales politiques et littéraires, La Revue des Deux Mondes... Dans le contexte idéologique des débats de l'époque, Goethe (écrit généralement Gœthe [sic!]) et son Faust sont invoqués de manière contradictoire comme facteur d'identité ou d'altérité, comme lien ou comme clivage entre les peuples. D'une part, on les loue comme preuve $\mathrm{du}$ cosmopolitisme et des échanges culturels entre nations (« les Leibnitz, les Schiller, les Gœthe étaient tout imprégnés de raison latine, de lyrisme anglais, de mesure, de bon sens français $\aleph^{14}$ ); mais on les dénonce d'autre part en tant qu'ils sont l'instrument de l'impérialisme intellectuel allemand, autrement dit de la Kultur :

Et le Faust, revu par la Kultur, présente, en effet, quelques-uns des caractères qui sont susceptibles de flatter un Allemand de 1915 : rêve d'orgueil et d'artifice, il réalise le premier essai du kolossal - Gœthe disait lui-même « incommensurable » et compose un monument qui n'est qu'une construction de l'esprit où le poète collabore avec l'archéologue et qu'on peut visiter sans recueillement. Mais, surtout, il produit une première ébauche du surhomme, surhomme dégoûté sans doute, ou plutôt Titan, de la race qui honore les géants en persiflant les dieux.

[...]

C'est le troisième Faust dont le scénario monstrueux se développe devant nos regards, un Faust plus national encore que les deux autres, qui rejoint les fables de sorcellerie et les marionnettes où le docteur, ayant vendu son âme à un vrai démon, entrâne dans sa chute Marguerite. Mais dans l'adaptation nouvelle, c'est l'Allemagne qui tient l'emploi de Gretchen ${ }^{15}$.

Moyennant une telle lecture de la fable faustienne, tout personnage risque d'être emporté dans le même discrédit. Ainsi, le traitement infligé à Faust et Marguerite dans le prologue de La Dernière Nuit participe du régime disqualifiant qui conduirait à traiter celle-ci de " vache sur champ d'azur $»^{16}$ comme à ranger celui-là parmi « les héros de la Kultur, les professors, les doktors, les intellectuels par excellence, les kantiens, les wagnériens ${ }^{17}$. Plus simplement encore tel dessin de C. Léandre, qui campe face à Faust un Méphistophélès portant croix pattée et casque à pointe, se trouve ainsi légendé : « FAUST.- Donne-moi la Jeunesse et l'Amour ! MÉPHISTO.- Donne-moi la Ruine et la Mort. $\aleph^{18}$ Il va sans dire que, dans le genre du pamphlet guerrier tel que Rostand le pratique dans Le Vol de la Marseillaise, le manichéisme fonctionne à plein rendement: c'est aussi comme Allemand que " Méphistophélès, dont le rire / Perdit quelques dents à Verdun, / Espère encor voir se détruire / Tous les hommes à cause d'un ${ }^{19}$. Une telle axiologie sous-tend évidemment le poème "Les Deux Propagandes", sous-titré "Préface pour un catalogue de livres de guerre ", où dès les premiers vers Rostand se figure en direct, en train de reprendre sa traduction de Faust :

«Où donc ai-je entendu déjà ce boniment?»

Murmurai-je.

On hurlait dans l'ombre, en allemand.

Soudain, je reconnus, à sa verve hideuse,

La Sorcière du Blocksberg, la Revendeuse

De Faust, qui proposait encore, à prix réduits, 
Sa camelote.

C'est du Gœthe. Je traduis.

"Allons, Messieurs », criait la Mégère éloquente,

«Choisissez ! Il n'est pas, dans toute la brocante,

Un pareil choix. Pas un de ces objets, Messieurs,

Qui n'ait fait quelque mal aux hommes sous les cieux!

Pas un poignard qui n'ait tué. Pas une buire... »

C'est du Gœthe toujours, je me borne à traduire.

«... Qui n'ait versé les plus corrosives liqueurs.

Pas un joyau dont l'or n'ait corrompu des cœurs.

Pas une épée, enfin, qui n'ait, avec vaillance,

Tranché d'un coup les nœuds sacrés d'une alliance,

Et frappé l'ennemi par derrière ${ }^{20}$ ! »

«Pas une épée, enfin, qui n'ait, avec vaillance, / Tranché d'un seul revers les nœuds d'une alliance / Ou bien qui n'ait frappé son homme dans le dos!... / Approchez! Achetez ! Choix de jolis cadeaux ! $"$ : ainsi s'achève dans son adaptation en vers la tirade de la "Sorcière revendeuse " (p.97), dont Rostand ne conserve ici à peu près qu'un distique. Cela s'explique certes par l'entrelacement des voix ; la rime buire : : traduire est à ce titre particulièrement savoureuse, où l'allemand Kelch, issu du latin calix, est rendu par buire, mot rare désignant une cruche, probablement d'origine francique. Mais c'est aussi et surtout qu'il veut donner à ces vers une dimension polémique :

\footnotetext{
Merci,

Gœthe! Nous nous étions demandé jusqu'ici

Où l'Empereur avait acheté son épée.

Ô Belgique, la nuit, par derrière, frappée,

Ce parjure est allé l'acheter au sabbat,

Le sabre avec lequel jamais il ne se bat ${ }^{21}$ !
}

À cette sorcière qui vante ensuite des livres plus terrifiants encore que toutes ces armes et ces instruments de mort, la suite du poème oppose une sirène, figure de vérité qui inspira la «Stella » d'Hugo, et qui se révèle pour finir être la bataille de la Marne et la fée Raison. Il n'y a que sa propagande de juste. Elle seule est pourvoyeuse des bons livres de guerre, «les livres de chevet / Qui donnent l'insomnie »"22. Opposé de manière topique à la clarté latine, c'est tout l'irrationnel germanique que personnifie cette bonimenteuse de bric-à-brac. Ainsi Goethe, dans l'axiologie mise en œuvre, rejoint-il plus ou moins le paradigme du Mal, en même temps que la sorcière brocanteuse (Trödelhexe) et son étalage d'horreurs, avec le personnel de la nuit de Walpurgis et tous les sabbats jamais organisés parmi les brumes germaniques.

\section{«Et pas une aile ! »}

Quasi contemporain de "Zone» d'Apollinaire, «Le Cantique de l'aile» ne fait que secondairement l'éloge des premiers aviateurs. Achevé en juillet 1911, ce long poème éponyme du recueil réitère l'une des composantes du mythe rostandien, dont le panache est la première et la plus retentissante illustration. « Remarquez du reste, écrit Pierre Citti, le rôle des volatiles chez Rostand, de L'Aiglon, avec la comparaison filée dans le titre des six actes, Chantecler, le Vol de la Marseillaise. Mythologie de la plume, au vol d'épée, métaphore surtout de la renaissance de la beauté à travers la poésie, du Phénix $»^{23}$. Nous allons voir que l'aile aussi devient pour Rostand un moyen de distinguer Faust et Don Juan, et de préférer l'un à l'autre. À la suite du «Cantique », le 
sonnet suivant vient nuancer la critique que Don Juan fait de Faust dans le prologue de La Dernière Nuit :

GETHE

«Et pas une aile !» dit le vieux Faust allemand

Lorsqu'il fuit, sur les monts, un dimanche de Pâques,

Ceux qui s'en vont, en bas, dans les gaités opaques,

Danser et chopiner théologalement.

« Pas une aile ! Oh ! monter ! à chaque battement,

Monter ! du fond sans fin des soirs ambrosiaques,

Voir décroître une terre où luisent quelques flaques,

Et boire la lumière à même un firmament !»

Ainsi, quand, pour lui faire une âme plus petite,

On n'avait pas encore usé de Marguerite,

Faust poussait ce grand cri sur la cime d'un mont.

Avant de soupirer : « Ma belle demoiselle»,

Faust avait rêvé d'être ou Latham ou Beaumont...

Car l'amour n'est jamais que le regret d'une aile ${ }^{24}$ !

19 Cinquième et avant-dernier d'une suite intitulée "Le Printemps de l'aile », il renvoie explicitement à l'épisode « Vor dem Tor » où Faust, cheminant en compagnie de Wagner, s'abandonne à la contemplation de la vallée qu'éclairent les derniers rayons du soleil. Si l'attaque très caractéristique est reprise à l'adaptation en vers « Et pas une aile, pas une aile, qui, du sol, / M'enlève, pour te suivre, ô Soleil, d'un long vol !» (p. 27), le second quatrain en soumet les onze vers suivants au régime de la condensation. Quant au sixain, il renvoie à l'épisode ultérieur de la rencontre entre Faust et Marguerite ("Strasse »). L'hémistiche au discours direct reprend la première réplique de Faust, ainsi traduite par Rostand : « Ma belle demoiselle, est-ce qu'on peut oser, / Son bras, et son escorte aussi, les proposer » (p. 64).

Commune à ce sonnet et aux deux textes déjà étudiés - la scène du pacte dans le prologue et «Les Deux Propagandes » - est la façon qu'a Rostand de mettre le mot «allemand(e)» à la rime, comme pour donner d'entrée au débat une dimension nationale. Au « vieux Faust allemand » sont ici opposés deux aviateurs français, Hubert Latham (1883-1912) et Jean-Louis Conneau dit André Beaumont (1880-1937). Si l'on ne peut y réduire son propos, Rostand reste à ce point hanté par le souci de faire l'unanimité qu'il multiplie pareilles concessions aux nationalistes. De manière peutêtre plus vivante et plus intime, il dépasse la conception de Faust comme repoussoir. Il le présente davantage comme un héros potentiel, accordé pour un temps à ses propres revendications ascensionnelles, mais qui y aurait ensuite renoncé pour rechercher l'amour de Marguerite. Si "l'amour n'est jamais que le regret d'une aile», c'est doublement vrai dans l'œuvre d'un idéaliste hanté par l'âme, conçue comme ce qui nous fait sortir de nous-même et nous force à nous dépasser ${ }^{25}$. En ce sens, Faust ne serait pas un anti-Don Juan, mais davantage un Don Juan qui aurait fait long feu, qui porterait comme Polichinelle le deuil de son âme. Du moins est-ce ainsi que nous comprenons cette réplique de Don Juan à la Marionnette du Diable :

Je suis le monstre avec une âme, Archange fauve

Qui laisse vivre encor son aile de déchu!

$\mathrm{Si}$, quand je passe, un souffle agite le fichu, 
C'est que je n'ai pas fait comme Polichinelle

Qui porte dans son dos le cercueil de son aile! (p. 47)

21 Le sonnet «Gœthe » pour lequel il sollicite Faust traduit les positions désabusées de Rostand sur le chapitre de l'amour. Après le prêt-à-porter sentimental des Romanesques, la fin'amor de La Princesse lointaine et l'amour religieux de La Samaritaine, les grandes œuvres renvoient davantage l'amour au chapitre des malentendus. L'amour n'est jamais qu'en partie l'âme qui fait «bondir au dessus de soi-même plus beau » (p. 122); ainsi Don Juan reconnaît-il avoir instrumentalisé ses conquêtes : « Oui ! Vous n'avez été que mes exaltatrices!» (id.). L'âme, l'aile, l'exaltation, l'inspiration, le souffle : autant de mots-clés pour la poésie et pour le théâtre de Rostand. Si Faust comme Polichinelle est un Don Juan avorté, tous trois ont en commun d'avoir connu, ne fût-ce qu'à titre provisoire ou de manière embryonnaire, l'orgueil diabolique d'égaler Dieu et celui non moins diabolique de se triompher du Diable. L'âme n'est-elle pas, en même temps que l'étincelle qui nous lie à Dieu, la distance qui nous en sépare?

Le théâtre de Rostand est un théâtre d'illusions. Conduit à dépouiller l'une après l'autre toutes ses écorces, confronté comme Chantecler à la mort de son rêve, il arrive à Don Juan ce qui arrive à l'Aiglon : ses ailes brisées, il ne reste plus de lui que le personnage public, ce que chacun voudra faire de lui. «Viens aux doigts des montreurs abdiquer ta personne » (p.139), intime le Diable à Don Juan ; "J'aurai tout manqué, même ma mort ", dit Cyrano. Il faut insister sur cette dynamique morbide et mortifère: le personnage de Rostand n'a de cesse qu'il ne soit délivré de ses illusions mais, loin de ressortir grandi de l'épreuve, il s'en trouve essoré, vidé. Comme si les illusions, le rêve étaient non l'enveloppe mais bien la substance, comme si ce qui abuse était en même temps ce qui soutient et qui donne la vie. «Ce maître des masques, à la fois acteur et metteur en scène qui s'était fabriqué son propre théâtre est voué à en devenir l'esclave $»^{26}$, écrit justement Carme Figuerola. Privé de tout principe créateur, Don Juan n'est plus que « ce beau costume vide / Où chacun glissera son rêve » (p. 137).

Sur la propension qu'a Rostand à nationaliser le mythe, il faut évidemment relire L'Aiglon. Dans un dialogue avec Prokesch (Acte IV, scène 4), l'Aiglon mélange les Don Juan, sans souci de l'anachronisme (si le Don Giovanni de Mozart est créé en 1789, le poème symphonique de Richard Strauss, de cent ans postérieur, ne saurait jouer que dans le cadre de la double destination) :

De la musique !... Et tu n'es plus, fils de César,

Qu'un Don Juan de Mozart !

Ricanant.

Pas même de Mozart :

De Strauss !

Il salue gravement Prokesch. Je vais valser.

Et pirouettant avec une gaieté désespérée. Il faut que je devienne

Inutile et charmant, comme un objet de Vienne !

Ce destin de bibelot qu'il envisage ironiquement comme échappatoire à sa cage dorée mais prussienne, n'est-ce pas déjà le sort d'un Don Juan devenant marionnette à la fin de La Dernière Nuit? Avec la Première Guerre mondiale, les choix ne feront que se confirmer. Si Rostand prend aussi souvent des distances par rapport à Faust, c'est à l'évidence par refus d'un mythe d'origine germanique. À propos de Faust justement, dans l'entretien de janvier 1913 déjà cité, il s'interroge : « [...] pourquoi aller chercher des idées de pièces en dehors de notre pays ? Rien répondra-t-il jamais mieux à notre 
tempérament, à notre goût, à notre éducation, qu'un de ces sujets que nous offre l'histoire de France? $\gg^{27}$ D'autant plus délibérément latin, Don Juan ne veut pour épitaphe que la mention des deux extrémités de son parcours amoureux : « Il naquit à Séville et mourut à Venise ! » (p. 26).

Nonobstant sa composante nationale, La Dernière Nuit de Don Juan dramatise le bilan du héros, d'une vie et d'une époque. Deux siècles plus tôt Molière abandonnait son personnage dans la force de l'âge, en pleine gloire : quoique condamné par le ciel et peut-être même parce qu'il l'est, son Dom Juan nous apparaît surtout comme un héros qu'auréolent son orgueil et ses défis. Rostand le saisit au vol et lui accorde avec dix ans de plus la possibilité de persévérer. Lorsque commence son poème dramatique, le système a certes démontré son efficacité puisque la liste est pleine, mais le pouvoir est usé, la machine à séduire désormais tourne à vide. C'est ce héros sur le retour qu'il choisit de remettre, non plus à la justice céleste, mais à une justice immanente. Instruit par le Diable, le procès sera celui de Don Juan par Don Juan. Pour Otto Rank qui achève avec elle son Don Juan, la pièce de Rostand représente l'aboutissement du processus de dégradation du mythe : Don Juan y « perd le dernier lambeau de son caractère héroïque [...] et c'est aussi sa fin dans la littérature ${ }^{28}$. Quelle que soit la validité de ce jugement quatre-vingt ans plus tard ${ }^{29}$, on ne peut ignorer la dimension intime du théatre voulu par Rostand: le procès du héros implique aussi le bilan personnel du poète bientôt cinquantenaire, qui depuis plus de dix ans se survit difficilement à lui-même. Enfin, audelà de ces deux bilans unis par la première personne, La Dernière Nuit de Don Juan pourrait instruire également le procès du théâtre en vers, parmi toutes ces vieilleries qui ne passeront pas le cap de la Première Guerre.

\section{NOTES}

1. Caroline de Margerie, Edmond Rostand ou le baiser de la gloire, Paris, Grasset, 1997, p. 213.

2. Pierre Citti, "Théâtre littéraire et théâtre à succès: la fausse réconciliation de Cyrano de Bergerac ", in Littérature et Nation $\mathrm{n}^{\circ} 5$ ( $2^{\mathrm{e}}$ série), mars 1991, Tours, Publication de l'Université F. Rabelais, p. 35.

3. Rosemonde Gérard, Edmond Rostand, Paris, Fasquelle Éditeurs, 1935, p. 81.

4. Ibid.

5. Lettre écrite de Lyon, le 14 mars 1903, éditée dans Histoires littéraires $n^{\circ} 38$, avril-mai-juin 2009, p. 70 .

6. Caroline de Margerie, Edmond Rostand..., op. cit., p. 189.

7. Lettre à Edmond Rostand du 12 février 1908, éditée dans Histoires littéraires, loc. cit., p. 80. Cette scène confronte Méphisto travesti en Faust et un écolier venu prendre conseil auprès du vieux maître.

8. Cité par Jacques Lorcey, Edmond Rostand, Atlantica-Séguier, coll. « Empreinte », 2004, p. 251.

9. Ibid., p. 253.

10. Faust de Goethe. Adaptation inédite par Edmond Rostand. Édition établie par Philippe Bulinge, Paris, Éditions théâtrales, 2007, p. 131. Toutes nos références en corps de texte seront faites à cette édition. 
11. Cité par Jacques Lorcey, loc. cit., p. 253.

12. Lettre de décembre 1913 à sa sœur Jeanne, cité par Émile Ripert, Edmond Rostand, sa vie et son œuvre, Paris, Hachette, 1968, p. 181.

13. Edmond Rostand, La Dernière Nuit de Don Juan, Paris, Fasquelle, 1921, p.10. Toutes nos références en corps de texte seront faites à cette édition, lisible sur Gallica.

14. George Fonsegrive, «"Kultur" et civilisation », La Revue hebdomadaire n ${ }^{\circ} 18$, mai 1915, p. 60.

15. Francis Chevassu, « Le troisième Faust », Le Figaro ${ }^{\circ} 35$ du jeudi 4 février 1915, p. 1.

16. Ibid.

17. Alexandre Hepp, «L'inutile Gœthe », Le Figaro n ${ }^{\circ} 46$ du 15 février 1917, p. 1.

18. "Nouvelle édition de Gœthe ", Le Gaulois n ${ }^{\circ} 13740$ du vendredi 28 mai 1915, p. 1.

19. Edmond Rostand, Le Vol de la Marseillaise, Paris, Fasquelle, 1919, p. 237.

20. Le Vol de la Marseillaise, op. cit., p. 241 et 242.

21. Ibid., p. 242

22. Ibid., p. 247.

23. Pierre Citti, «Théâtre littéraire et théâtre à succès... ", art. cit., p. 33.

24. Edmond Rostand, Le Cantique de l'aile, Paris, Fasquelle, 1922, p. 24.

25. Pour les développements qui suivent, voir B. Degott, « Mourir (et renaître) pour des idées. Le théâtre en vers de Rostand ", in Mourir pour des idées, textes réunis et présentés par Caroline Cazanave et France Marchal-Ninosque, Besançon, pufc, 2008, p. 471-490.

26. Carme Figuerola, "La Dernière Nuit de Don Juan ou le chant du cygne », in Edmond Rostand: renaissance d'une œuvre. Textes rassemblés par Guy Lavorel et Philippe Bulinge, Université JeanMoulin. Lyon 3, C.E.D.I.C., 2007, p. 165.

27. Cité par Jacques Lorcey, Edmond Rostand, loc. cit., p. 250.

28. Otto Rank, Don Juan et Le Double [Don Juan und Der Doppelgänger, 1932], Petite Bibliothèque Payot, 2001, p. 225.

29. Voir Géraldine Vogel, "Démythification de Don Juan dans La Dernière Nuit de Don Juan d'Edmond Rostand », Le Paon d'Héra n ${ }^{\circ}$ 7, décembre 2010.

\section{INDEX}

oeuvrecitee Cyrano de Bergerac, Don Juan

Palavras-chave : verso, teatro

Palabras claves : verso, teatro

Keywords : verse, theater

Mots-clés : vers, théâtre

\section{AUTEURS}

\section{BERTRAND DEGOTT}

Université de Franche-Comté

Maître de conférences en littérature française 